\title{
VACCINATION AND THE EL TOR VIBRIO A BRIEF REVIEW
}

\author{
Lieutenant-Colonel J. E. NOBLE, \\ M.C.Path., M.R.C.S., L.R.C.P., D.T.M. \& H., R.A.M.C. \\ The David Bruce Laboratories
}

\section{Introduction}

DURING the past 25 years numerous outbreaks of a cholera-like disease, usually referred to as paracholera, have occurred in Indonesia (Pollitzer 1959). This disease is clinically indistinguishable from classical cholera and a redefinition, by World Health Organization, of "cholera" now includes cholera El Tor (World Health Organization 1962).

In the last 100 years cholera has disappeared from Europe and Africa and gradually receded to two localized endemic areas which by 1960 consisted of the Rivers Ganges and Brahmaputra delta for Vibrio cholerae and the Island of Celebes for Vibrio el tor (World Health Organization 1965).

From 1961 onwards cholera El Tor has rapidly increased its geographic range at first eastwards and later westwards until by 1965 it has reached the very threshold of Europe (Figure 1). Published outbreaks include:- India, Thailand, the Philippines, Indonesia and Sarawak (Felsenfeld et al 1962); Hong Kong (Mukerjee and Roy 1962); Malaysia (Noble 1964a); Burma and East Pakistan (Mukerjee et al 1965); East and West Pakistan and India, Philippines, Borneo, South Korea (Mukerjee et al 1965). During 1965 outbreaks occurred in Pakistan, India, Afghanistan, Persia and South U.S.S.R. (British Medical Journal 1965).

Investigations into some of these outbreaks revealed two alarming features; firstly that, in the areas where it occurred, infection with $V$. el tor was commonplace with comparatively few typical cases of cholera and secondly, there were many carriers, some of whom excreted the organism for up to three years. Convalescents, normally stoolnegative, when purged or when suffering from intercurrent diarrhoea, were shown to excrete $V$. el tor.

There appears to be a significant risk that in the near future expansion of cholera El Tor could continue, due, not only to pilgrimages and migrations in the Middle East where sanitation standards are not as effective as in Europe, but also to modern rapid transport methods and immigration from the East, giving rise to family infections and localized outbreaks in Europe itself.

\section{Vibrio el tor}

Paracholera is caused by Vibrio el tor, an organism which has many characteristics in common with Vibrio cholerae, the causative organism of classical asiatic cholera. Both organisms have identical morphology and cultural characteristics, belong to the Heiberg fermentation group I and share common $O$ group antigens.

A number of differences between the two vibrios have been described; haemolytic 


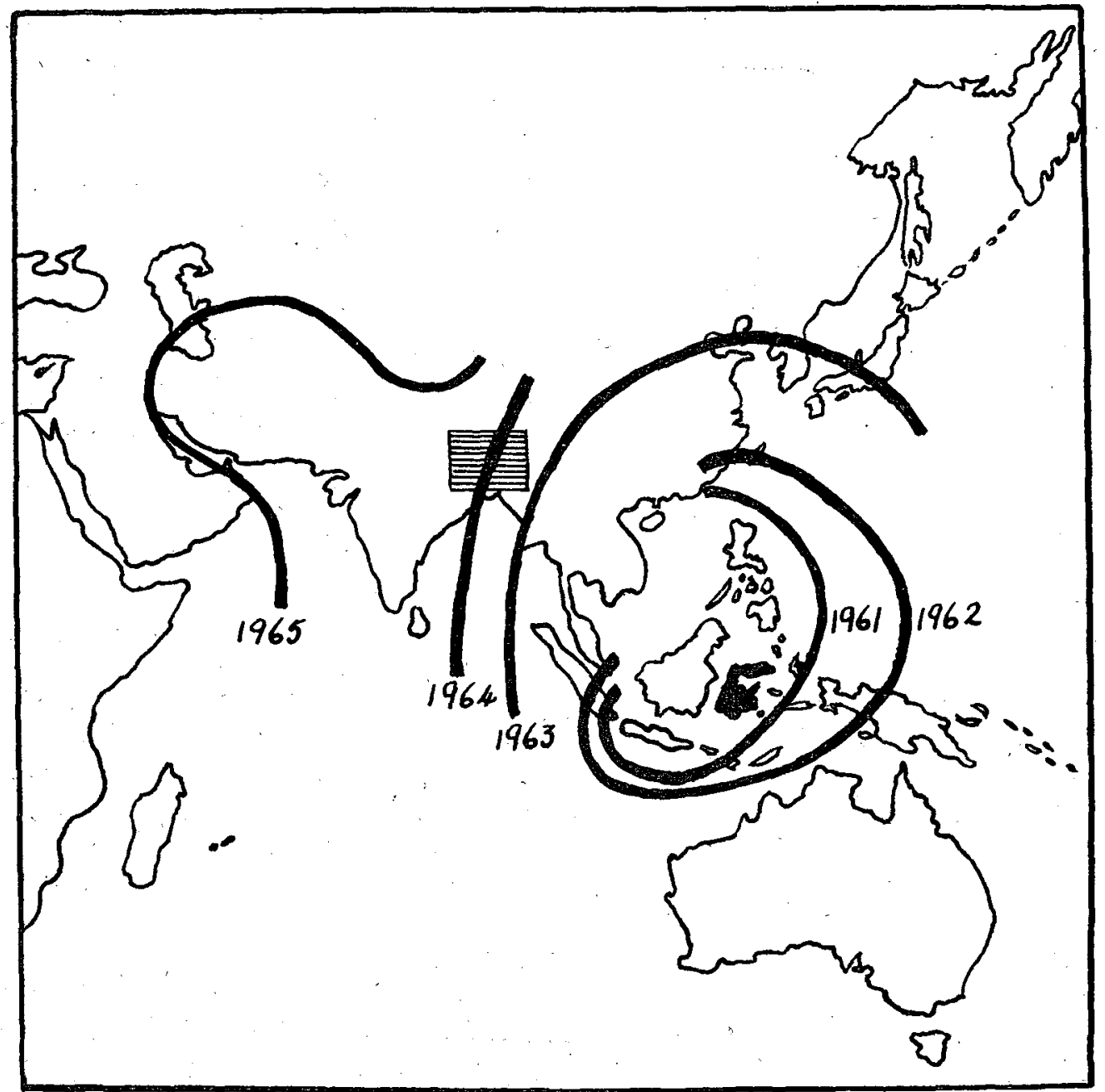

Fugure 1.

The hatched area represents the endemic area for classical cholera and the black island (Celebes) represents the endemic area for cholera El Tor in 1960, adapted from W.H.O. Chronicle (1966), 20, 263.

activity, toxin, protein composition, sublimate precipitation, soda agglutination, Voges Proskauer reaction and heat and chloroform inactivation of agglutinability (Pollitzer 1959). Bacteriophage typing (Mukerjee 1961), haemolysin testing either as recommended by Feeley and Pittman (1963) or chicken red-cell agglutination test (Finkelstein and Mukerjee 1963), and mouse toxicity (Noble 1964a) provide concise methods of differentiating the two vibrios. 
against cholera was Ferran in 1885. During the 1884 outbreak of cholera in Spain, he was able to demonstrate that guinea-pigs, which survived inoculation with a bile broth culture of live cholera vibrios, were resistant to the administration of further doses lethal to unprotected animals. Gamaleia (1888) showed that it was possible to protect guinea-pigs against lethal doses of $V$. cholerae not only with live organisms of reduced virulence, but also with organisms killed by heating at $120^{\circ} \mathrm{C}$. Many workers continued to experiment with living vaccines until Kolle (1896), using a cholera vaccine prepared by heating a suspension of cholera culture at $56^{\circ} \mathrm{C}$. for one hour, showed that the bactericidal properties of sera obtained from human volunteers were as high, if not higher, ten days after one injection of this vaccine than those obtained after a course of three injections of live cholera vaccine.

In the years that followed many workers continued experimenting with one form of cholera vaccination or another. These experiments included:- live vaccines with cholera strains of decreased virulence; inactivated vaccines in which the organisms were killed by various methods including heat, chloroform, phenol and formalin; whole fluid-culture vaccines in which the vibrios were grown on casein hydrolysate medium, killed by formolization and injected in entirety; vaccination with supernatants, filtrates and autolysates of cholera cultures; cholera formol toxoids; and attempts at vaccination by both the intranasal and oral routes. The outcome of all this work was that, by the 1950's, a series of almost universally acceptable standards for the production of a heat-killed phenol-preserved divalent cholera vaccine containing 8,000 million organisms per $\mathrm{ml}$. had been evolved. These standards, which include the nature, virulence and serotype of the vibrios, methods of culture and inactivation, and recommended techniques for assaying the resultant cholera vaccine, were laid down by World Health Organization (1959).

Although cholera vaccine was the first bacterial vaccine to be introduced for the control of communicable disease in man no proper evaluation has been made in a strictly controlled field trial until recently. Indeed, although it has been widely used for 80 years there has been no proof as to its efficacy. Under the auspices of S.E.A.T.O. Oseasohn, Benenson and Fahimuddin (1965) carried out a field trial in rural East Pakistan using a classical divalent cholera vaccine described in detail by Feeley and Pittman (1965). These authors state: "During the first year of a double-field trial of a cholera vaccine in an endemic area, the incidence of diarrhoea associated with $V$. cholerce was 6.1 per 1,000 among controls given a T.A.B. vaccine, and 1.7 per 1,000 amongst subjects given commercial cholera vaccine of high potency." It would thus appear that the efficacy of cholera vaccine in protecting against $V$. cholerae has at last been proven.

In recent years several authors have advocated the administration of cholera vaccine by the intradermal route. Singer, Wei and Hoa (1948) found that better antibody production was obtained by this method of vaccination and Panja and Das (1947), whilst noting the economy of materials and negligible reactions resulting from intradermal cholera vaccination, considered the method impracticable in mass inoculation programmes. Noble (1964b) found that the protection afforded by cholera vaccine administered by the intradermal route and demonstrated by active and passive mouse-protection tests, active guinea-pig tests, and antibody responses in both rabbit and man, was excellent and equal to that given by subcutaneous inoculations. He found that intradermal inoculation greatly reduced the incidence of reactions to cholera vaccine and was a practical proposition when the rapid immunization of large numbers was necessary. 


\section{Vaccination and the El Tor vibrio}

Mukerjee and Guha Roy (1962), Felsenfeld et al (1962) and the author of a leading article in the British Medical Journal (1962) have suggested that classical cholera vaccine may be of doubtful value for mass immunization against outbreaks of El Tor cholera. This view has again been expressed by the author of a leading article in the British Medical Journal (1965) and by Mukerjee et al (1965).

Feeley and Pittman (1963), Vella (1963) and Noble (1964a) have shown that classical cholera vaccines are capable of protecting mice against $V$. el tor to the same degree as against $V$. cholerae.

Active and passive protection tests were described by Noble (1965) in which classical cholera vaccine protected laboratory animals as effectively against challenge with the El Tor vibrio as against challenge with $V$. cholerae. Furthermore in these experiments the incorporation of $V$. el tor in cholera vaccine gave no improvement in the protection afforded to animals challenged with that organism.

In 1965 a field-trial was carried out by the Philippines Cholera Committee. In this trial a classical $V$. cholerae vaccine, an oil-adjuvant vaccine and a $V$. el tor vaccine were compared in a controlled field trial on some 584,000 people in an endemic cholera El Tor area in the Philippines. It was shown that cholera vaccines gave moderate protection of short duration. The overall carrier rate was reduced from 12.4 per cent in the controls to 8.2 per cent in vaccinated individuals. Injection of a single dose of vaccine prepared from either $V$. cholerae or $V$. el tor gave over 50 per cent protection for the first three to four months. This confirmed the mouse-protection experiments cited above in which cholera vaccination was followed by challenge at seven to ten days.

The immunity conferred by $V$. cholerae vaccine rapidly declined after four months whereas $V$. el tor vaccine appeared to give protection for six months before its effectiveness declined. However when all the figures were subsequently analysed the apparent advantage of the El Tor vaccine was found not to be statistically significant. An oiladjuvant vaccine prepared from $V$. cholerae conferred an increasing degree of protection of longer duration but owing to severe vaccination reactions its use could not be recommended.

In 1964 and 1965 two further cholera vaccine trials, sponsored by the World Health Organization, have taken place in Calcutta where the El Tor vibrio is now the predominant epidemic strain. Both these trials support the view that conventional cholera vaccine gives moderate protection against the $\mathrm{El}$ Tor vibrio, that this protection is of short duration and that its effectiveness has waned by six months.

\section{Conclusion}

It is not generally realized that the last notifications for cholera in Europe were as recent as: Greece 1922, Roumania 1922, Spain and Germany 1921, Italy 1916 and England 1911 (Pollitzer 1959).

The increase in the geographic range of cholera which has occurred during the last five years very closely resembles that shown in the early part of the most recent sixth cholera pandemic of 1899 to 1922 as is shown in table 1. For reasons which are stated earlier there, is a significant risk that cholera could spread further West and involve some parts of Europe. 


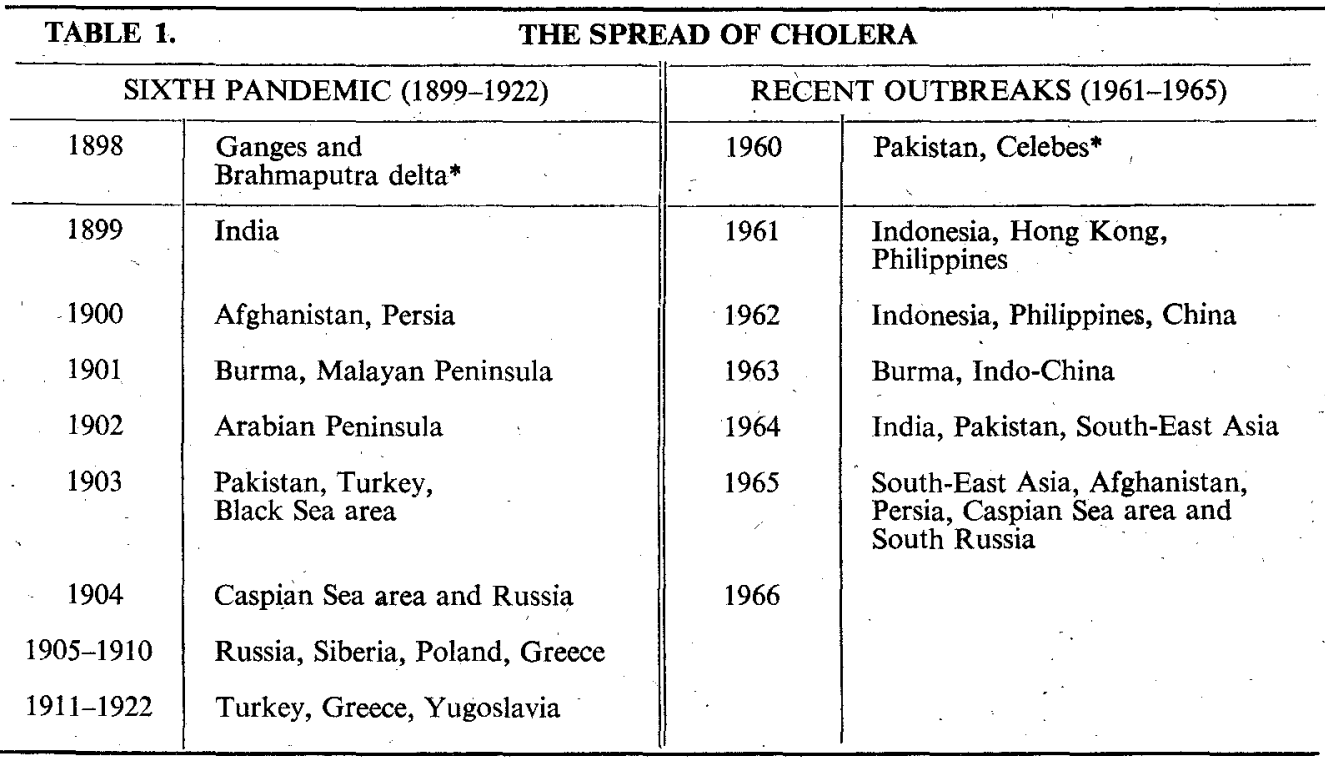

*These are the endemic foci of origin of the outbreaks listed below them.

In an article, Mukerjee et al (1965) have suggested that the $V$. el tor appears to inhibit $V$. cholerae both in vitro and in vivo and that for this reason the El Tor vibrio is now the principle causative organism of cholera in most areas.

These facts have given rise to a renewed interest in cholera vaccines and to doubts as to whether the conventional divalent cholera vaccines would protect against $V$. el tor.

Although recent field trials have confirmed that cholera vaccines have a valuable part to play in the protection of the individual against cholera, the principal defence against this disease must still be by detection of cases and carriers, by the highest standards of hygiene and by health education. There is no doubt that conventional cholera vaccines of high potency protect as well against the El Tor vibrio as against $V$. cholerae. This protection is short lived and reinforcement of immunity should take place at intervals of not less than six months. For those in continued intimate contact with cholera an interval as short as three months should be considered if the maximum benefit is to be derived from vaccination.

Extensive research is continuing in America, India, Pakistan and in/this country at the David Bruce Laboratories and elsewhere with conventional cholera vaccines, El Tor vaccines and trivalent vaccines containing both $V$. cholerae and $V$. el tor. It is highly probable that in the near future a more effective vaccine of higher potency than those at present in use, yet remaining as free from severe reactions, will be found, in order better to protect the individual against cholera regardless of whether it is caused by $V$. cholerae or $V$. el tor.

British Medical Journal (1965). (ii). 832.

\section{REFERENCES}

Feeley, J. C. and Pittman, M. (1963). Bull. Wld. Hlth. Org. 28, 347.

FEeley, J. C. and PitTMAN, M. (1965). Lancet (i), 449.

Felsenfeld, O., MưkerJeE, S, and NASUnIYA, N. (1962), J. Trop. Med. Hyg. 65, 200. 
FERRAN, J. (1885). Siglo. med. 32, 480.

Finkelstein, R. and MuKerJeE, S. (1963). Proc. Soc. Exp. Biol. and Med. 112, 355.

Gamaleia, M. N. (1888). C.R. Acad. Sci. (Paris). 107, 432.

Kolle, W. (1896). Zbl. Bkt. I. Abt. Orig. 19, 97.

Leading Article (1962). Brit. med. J. (i), 701.

MUKERJEe, S. (1961). J. Hyg. Camb. 59, 109.

MuKERJEE, S. and GuHA RoY, U. K. (1962). Brit. med. J. (i), 685

Mukerjee, S., Basu, S. and Bhattacharya, P. (1965). Brit. med. J. (ii), 837.

NoBLE, J. E. (1964a). J. roy. Army med. Cps. 110, 15.

NOBLE, J. E. (1964b). J. Hyg. Camb. 62, 11.

Noble, J. E. (1965). J. Path. Bact. 89, 543.

Oseasohn, R. O., Bentenson, A. S. and Fahimuddin, M. (1965). Lancet (i), 450.

PANJA, G. and DAS, N. W. (1947). Indian J. med. Res. 35, 3.

Philippines Cholera Committee (1965). Bul. Wld. Hlth. Org. 32, 603.

PollitZer, R. (1959). Cholera. Wld. Hlth. Org. Monogr. Ser. No. 43.

Singer, E., WeI, S. H. and HOA, S. H. (1948). J. Immunol. 60, 181.

Vella, E. E. (1963). Brit: med. J. (i), 1203.

World Health Organization (1959), Wld. Hlth. Org. Tech. Rep. Ser. No. 179, p. 31.

World Health Organization (1962). Quarantine and Prevention of Disease (Amendment) Ordonance (1962).

World Health Organization (1965). Cholera Information No. 5.

World Health Organization Chronicle (1966), 20, 263.

\section{LETTERS TO THE EDITOR}

\section{VISCERAL LEISHMANIASIS IN THE ADEN PROTECTORATE}

From: Lieutenant-Colonel C. G. B. DOWNIE, B.Sc., M.B., M.R.C.P., M.R.C.P.E., D.T.M.\&H. SIR-I should like to congratulate Major Ian Michie on his interesting article on this subject which appeared in the January 1966 issue. He reports the first four cases of visceral leishmaniasis in troops operating in the Aden Protectorate. I can add a fifth case from the other side of the world.

A 23 year old Lance Corporal in the K.O.S.B. was admitted to B.M.H. Hong Kong on 23rd June, 1965 , for investigation of a fever of a few days duration. He had a basal pneumonia which cleared with tetracycline therapy but his temperature remained elevated for four weeks during which he remained well and cheerful, sometimes eating huge meals with a smile on his face and a pyrexia of $105^{\circ}$. During this time his white cell count remained low, his spleen slowly enlarged, and he became progiessively anaemic, down to a level of 7.6 grams per cent. He also developed hyperglobulinaemia. Since we knew he had been in Aden from May to October 1964 leishmaniasis was suspected early, but repeated blood examinations, and marrow and liver biopsies failed to reveal the parasite. However on 19th July treatment with sodium stibogluconate (Pentostam) was started. His fever immediately fell and all abnormalities rapidly disappeared. I have no doubt on clinical grounds that he was suffering from visceral leishmaniasis. He was later fit enough to go with his battalion to Borneo.

He was mostly on convoy guard duty between ADEN and THUMIAR during his stay in Aden. However in July 1964 he spent 3 weeks on patrol in the Radfan area often staying near small villages and lying on the ground on sand or grass. He also did one convoy up the DHALA road while temporarily attached to the Royal Scots.

AMD Stats should now be able to produce total numbers of cases of leishmaniasis occurring in British troops stationed in Aden. This case emphasised once again the importance of taking a careful and detailed history.

British Military Hospital,

Hong Kong,

B.F.P.O. 1.

I am, etc.,

C. G. B. DOWNIE.

16th June, 1966.

\section{MARATHON AND THE W.B.G.T. STRESS INDEX}

From: Colonel F. G. NEILD, M.R.C.S., L.R.C.P., D.P.H., D.I.H., late R.A.M.C.

SiR-While motoring out here recently, MARATHON, just outside ATHENS, was passed with the shade temperature in the high eighties. This made one wonder whether the experts had ever considered what the probable W.B.G.T. Stress Index was when Phaedippides ran the first Marathon on the (?) 21st September, 491 B.C.

H.Q.,

UNFICYP

I am, etc.,

F. G. NEILD.

26th July, 1966.

NICOSIA;

B.F.P.O. 667. 